This article has been retracted: please see INNOVATIONS in pharmacy retraction policy (https://pubs.lib.umn.edu/index.php/innovations/policies). This article has been retracted by the Editor and Publisher due to the inappropriate use of previously published work. 


\section{Extemporaneous Compounding: Selective Pharmacists with Separate Skill}

AK Mohiuddin, Assistant Professor

Department of Pharmacy, World University of Bangladesh, Bangladesh

\section{Abstract}

Extemporaneous compounding takes place in community and hospital pharmacies. There are usually specialist compounding pharmacies in major towns and cities, but any pharmacy may undertake compounding as long as they have appropriate facilities according to state-based legislation (e.g. allocated clean bench, specific compounding equipment). Although development is a continuous process, companies are customizing features to meet the majority of patient needs, but the very nature of the process cannot meet all patient needs. The risk-benefit ratio of using traditionally compounded medicines is favorable for patients who require specialized medications that are not commercially available, as they would otherwise not have accescto suitable treatment. However, if an FDA-approved drug is commercially available, the use of an unapproved compounded ag cor additional risk with no commensurate benefit. Published reports of independent testing by the FDA, state agench and oth sonsistently show that compounded drugs fail to meet specifications at a considerably higher rate than FDA-appro d drus Comp inded sterile preparations pose the additional risk of microbial contamination to patients. In the last 11 years, th ce sepgrate ingitis outbreaks have been traced to purportedly 'sterile' steroid injections contaminated with fungus or bacteria, hich ere made by compounding pharmacies. The 2012 outbreak has resulted in intense scrutiny of pharmacy compounding pras ces an ncreas recognition of the need to ensure that compounding is limited to appropriate circumstances.

Keywords: Pharmacy Practice; Drugs; Dosage Forms; Compounding; Eg Phar cropeia

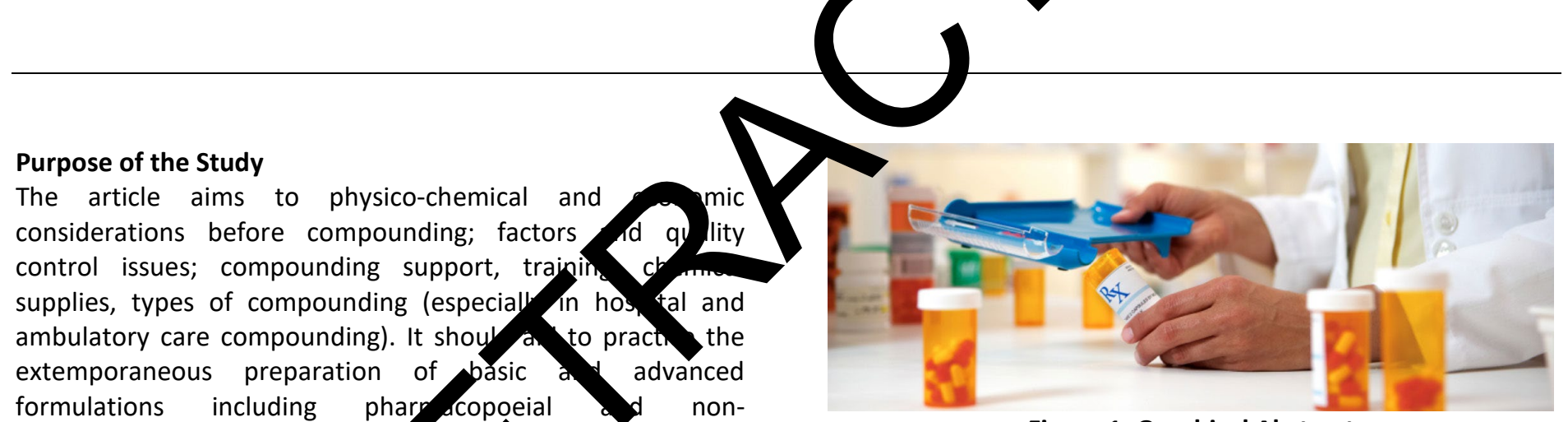
formulations including phar acopoeial od nonpharmacopoeial formulations ncoured in pharmacy practice, together with reguisito cumertion, labeling, packaging and counseling equ emen $A$ ng with the they have to study the ralysis of formulations and their components and relate to truclinical performance of medicines. This will help om to investigate, evaluate and report the physical characten tics of formulations including release kinetics and relate these to quality control and preformulation requirements; relate the application of quality control, quality assurance and the principles of good manufacturing practice to regulation of medicine production in home and abroad.

Corresponding author: AK Mohiuddin, Assistant Professor Department of Pharmacy, World University of Bangladesh 151/8, Green Road, Dhanmondi, Dhaka - 1205, Bangladesh Phone: +8801716477485; Email: trymohi@gmail.com

Article Highlights:

1. An estimate by FDA that $1-10 \%$ of all prescriptions require compounding was not supported by evidence data.

2. In 2006, the FDA conducted a limited survey of compounded drugs revealed failure rate of $33 \%$.

3. In 2001, a Kansas City-based pharmacist was discovered to have adulterated 72 different drugs, including many oncology medications, to increase profits.

4. Over the period 2008-2010, the Texas State Board of Pharmacy found an overall potency failure rate of $23 \%$ for compounded drugs

5. Methylprednisolone injections from a single compounding pharmacy in the USA resulted in 137 cases of Aspergillus fumigatus meningitis and 12 deaths.

6. Even where a given formulation has been shown to achieve suitable physical, chemical and microbiological stability, the bioavailability and palatability of the preparation may be unproven. 
7. Ingredients, intended use, dosage, and method of administration, education, skill and expertise; proper equipment, supplies, chemicals the guidelines delineated in US pharmacopeia, necessary calculations and documentation; expected duration of therapy, physicochemical compatibility are the major considerations before compounding.

8. Quality must be built-in to the preparation from the beginning steps to evaluating the final preparation.

9. The use of an alternative route of administration (use of the rectal rather than the oral route) could also be considered if an appropriate formulation is available.

\section{Introduction}

The FDA defines traditional pharmacy compounding as the combining, mixing, or altering of ingredients to create a customized medication for an individual patient in response to a licensed practitioner's prescription. The NABP further describes compounding as the result of a practitioner's prescription drug order based on the practitioner/patient/pharmacist relationship in the course of professional practice. Traditional pharmacy compounding plays a valuable role in providing access to medications for individuals with unique medical needs, which cannot be met with a commercially available product. For instance, a prescriber may request that a pharmacist compound a suspension for a pediatric or geriatric patient unable to swallo a medication in its commercially available form. In tradition pharmacy compounding, an individualized medicine prepared at the request of a prescriber on a smal cale obvious that large scale manufacturers have ery li itad opportunity to customize dosage strength and $\mathrm{sas}$ ms for a customer segment that is not substantial ig enoug vue to economies of scale concept of bus, ess. nother Major difference is that compounding phary acies are no required to report adverse events to the $E^{\prime}$, whareas adverse event reporting is mandatory for ma fact ers of FDA-regulated medications. Thus, ady osociated with compounded drugs may diffi It to at, particularly if the affected patients are 5 tely $c$ rand in different geographic areas. Registered and skil oharmacists in practicing their art of compounding fills in this $\mathrm{g}$ to meet individualized needs. Compounding has always been a basic part of pharmacy practice; the drugs, dosage forms, and equipment or techniques used are the variables. Pharmacists have unique knowledge and skills and are not duplicated by any other profession. Pharmacy activities to individualize patient therapy include compounding and clinical functions. Either function in the absence of the other results in placing pharmacy in a vulnerable position. It is important to entrust a pharmacist's expertise to adjust dosage quantities, frequencies, and even dosage forms to enhance compliance. Pharmacy is a complex mixture of different practices and practice sites. No longer is pharmacy simply community pharmacy or hospital pharmacy. Pharmacy is diverse and offers many opportunities for those willing to look around, find their niche and practice pharmacy to meet the needs of their own community of patients. Most compounding pharmacists appear to be interested and excited about their practices. In fact, many pharmacists intimately involved in pharmaceutical care have now realized the importance of providing individualized patient care through the preparation of patient-specific products. Compounding pharmacy is not for everyone, but as it grows, it will provide an increasingly significant number of pharmacists the excitement and fulfillment of using their innovative and creative skills to solve patient problems. All components of a compounded product, that is the active ingredient and the excipients, are subject to quality standards set out in the Therapeutic Goods Act 1989, and are sourced from compounding supply companies that undertake rigorous quality-assurance testing.

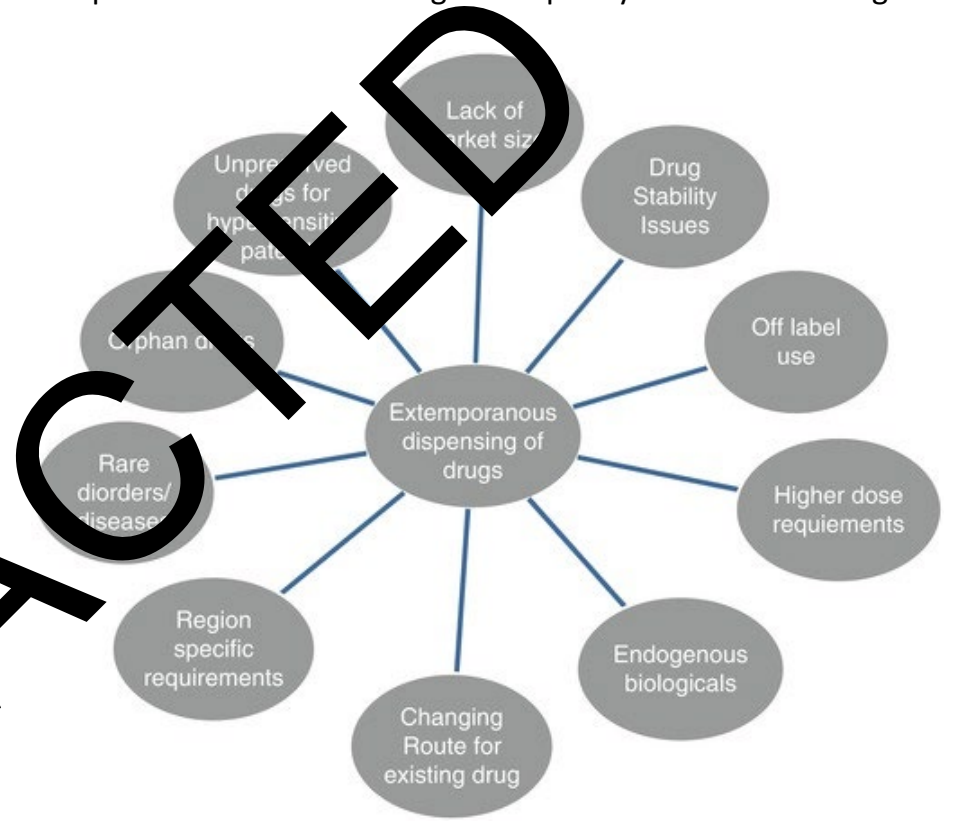

Figure 2. Rationale of Extemporaneous compounding (Source: Velpandian T., Das U.S. (2016) Extemporaneously Used Drug Formulations for Ocular Emergencies.

In: Velpandian T. (eds) Pharmacology of Ocular

Therapeutics. Adis, Cham

\section{Rationale of Extemporaneous Compounding}

An estimate by FDA that $1-10 \%$ of all prescriptions require compounding was not supported by evidence data. EP strategies in clinical studies requires 'fit-for-purpose' formulation design and preparation processes, as well as administration procedures that are safe, flexible, cost-effective, and simple to adapt by a compounding pharmacist at the clinical site. Many commercially available medications are not manufactured in suitable dosage forms for these special populations, e.g., liquid formulations for children and those who may be unable to swallow tablets. Additionally, when drug shortages occur or for the treatment of rare diseases, compounded products have a place in practice. Examples of altered drug products include topical ointments, eye drops, solid or liquid oral dosage forms, and intravenous infusions. Compounding also has a place in veterinary practice, where 
medicines may be altered to suit drug absorption, metabolism, and swallowing abilities of different species. The use of smallscale compounded medications carries certain risks, as they are not assessed for product quality, stability, or efficacy. The importance of a collaborative, team-based approach to patient care and safety has been well established within the health field. The requirement for alternative medications and dosage forms may be determined by the patient's general practitioner who prescribes the medication or other allied health professionals: a speech pathologist who identifies a swallowing difficulty, a physiotherapist applying ultrasound, which may interfere with transdermal drug delivery, or an occupational therapist identifying that a patient is unable to physically manipulate their current medication or its packaging. This highlights the importance for other members of the health care teams to have knowledge and understanding of compounded medications. Among elderly patients, the incidence of dysphagia is reportedly as high as $7 \%-13 \%$, making utilization of available oral dosage formulations unfeasible or unsafe. Similarly, Canadian hospital pharmacists often encounter patients with oral, neck, or gastric cancer, which may make commercial solid dosage formulations impossible to use. Based on pharmacists' perspective, using more extemporaneous prepared products could also make sense economically. 'The significant therapeutic improvement and some cost savings may be gained' by the rational use of extemporaneously prepared medicines. Pharmacists that did not provie compounding service cited by the main reason as not receivin prescriptions that required compounding $(63.6 \%$ compounder pharmacists). In about $33 \%$ of the co this reason concerned a strictly defined pha issues. Some researchers conclusively fid that $73.2 \%$ pharmacists reported their goal in provid g fun harmace utical care to their patients was the most iportant $n$ ivator. The main reasons for the decision to $y$ ovide ampounarng service were want to provide full pha ace clal care to patients (73.8\% of compounder phar vist) a resp ding to requests by prescribers. Most ch imur y pho acists (nearly 70\%) accepted compoundin as a manent of pharmaceutical care. In most of the studie najor reasons stated by physicians for prescribing extemporan us products were that they worked well for certain conditions and that the product was not available commercially [1-7].

\section{Evaluation of the Need}

- Soluble or dispersible tablets may be a useful and convenient alternative to the preparation of liquid extemporaneous products. Some tablets can be dispersed or crushed and information on this aspect can be obtained from the Medicines Information Section within the healthcare setting. In this case, the dose should be prepared and administered immediately. In general, compressed tablets or tablets which are scored or just film coated can be crushed whereas modified release tablets cannot.
- If a particular medicine is not available as a liquid formulation, another medicine from the same therapeutic classification may well be used, such as the use of a less potent steroid rather than diluting a potent one.

- Using a suitable preparation intended for a different route of administration, for example, using an injectable solution orally.

- Use of a 'specials' preparation manufactured in licensed premises (Specials are medicines made in larger volumes by a licensed manufacturer) [7].

\section{Considerations before compounding}

- Commercially availability of drug in dosage form, strength, and pac

- Ingredients, in nded use dosage, and method of administration co cern

- Educatio skill and per ce to drug compounding

- Prope equin Rent, unplies, chemicals and the gui elin uneate in us pharmacopeia

- be fiterna which the patient will receive a Safety the compounded product

Patient necessary storage facility, if required,

- Nessary calculations to prepare the product

- N cessary documentation to complete preparation iterature reference that might provide information on use, preparation, stability, administration

Expected duration of therapy

- Some basic quality control to check the product prior to dispensing (e.g., capsule weight variation, $\mathrm{pH}$, visual observations)

- Ingredient identity, quality, and purity

- Corrective methods

- Physico-chemical incompatibilities $[5,6]$

Economic Considerations: The most significant factor which limits prescribing of compounded medicines was that patients did not want products due to these products were not covered by the national insurance. Furthermore, for doctors not prescribing these products, the major barrier to prescribing was their lack of knowledge of the products. Most responding doctors do not prescribe medications that require compounding because they lack trust in the quality of the compounded formulations. However, most of the doctors expressed a desire to learn more as shown in having access to information about the usefulness of extemporaneous products. There are at least two different economic considerations in making the decision to compound prescriptions; these include (a) pharmacist compensation and (b) health-care costs. The pricing of a compounded prescription should include consideration for pharmacodynamic and pharmacotherapeutic decision making, formulation expertise, time, and reimbursement of materials. Compounding prescriptions can be attractive professionally and financially. Compounding prescriptions can be a way of lowering the cost of drug therapy. 
In some cases, it is less expensive for the pharmacist to prepare a specific prescription for the patient, which may mean the difference between the patient actually obtaining the drug or doing without it. If compounding a prescription results in a patient being able to afford the drug therapy, it must be considered. An interesting example involves the drug 4aminopyridine. Although physicians had been prescribing unapproved versions of the drug for up to 20 years, it was not until the drug was studied systematically that rare seizures were discovered as a potential side effect. In this case, the medical profession pushed for an approved version to be marketed, rationalizing that if a seizure occurred in the context of a patient taking an FDA-approved alternative [4-10].

\section{Compounding Factors}

Stability: One key factor in compounding prescriptions is stability. The more common types of stability of which compounding pharmacists should be aware include chemical, physical, and microbiological. Whereas commercially manufactured products are required to possess an expiration date, compounded products are assigned a beyond-use date. There are numerous sources of information that can be used for determining an appropriate beyond-use date, such as chemical companies, manufacturers literature, laboratory data, journals, and published books on the subject. Generally, most pharmacists prepare or dispense small quantities of compounded products; recommend storage at room, cool, cold temperatures; and use a conservative beyond-use date For nonaqueous liquids and solid formulations (for which the manufactured drug product is the source of active ingredient) - The beyond-use date is not later than $25 \%$ of the time remaining until the product's expiration date or 6 months, whichever is earlier. A USP or NF substance is the source of active ingredient- The beyond-use date is not later than 6 months. For water-containing formulations (prepared from ingredients in solid form)-The beyond-use date is not later than 14 days when stored at cold temperatures. For all other formulations-The beyond-use date is not later than the intended duration of therapy or 30 days, whichever is earlier. These beyond-use date limits may be exceeded when there is supporting valid scientific stability information that is directly applicable to the specific preparation (i.e., the same drug concentration range, $\mathrm{pH}$, excipients, vehicle, water content). For example, active ingred ntaining an ester functional group, such as aspirip and pen lins, are susceptible to breakdown by hydrolysis, vhile tho containing aldehyde or hydroxyl groups, $\mathrm{f}$ ch as tes ter e and dopamine, undergo oxidative deco ositio A 10 increase in temperature can result in a 2 -fo, ster rat of degradation. The instability of dapsone spension vre ared in Oral Mix and stored at $25^{\circ} \mathrm{C}$ may b exp, ned by a vaillard reaction between the sucrose in theyehicle a. the amino groups of the dapsone. The results this study have demonstrated the stability, for up to 90 days, ff dapson suspensions ( $2 \mathrm{mg} / \mathrm{mL}$ ) prepared from commercial blets in $\mathrm{al} \mathrm{Mix} \mathrm{SF}$ and stored at $5^{\circ} \mathrm{C}$ and $25^{\circ} \mathrm{C}$ or prepared in Or an: and stored at $5^{\circ} \mathrm{C}$, in amber plastic bottles and amber -sstic syringes. These suspensions should be shaken before use [2],[5],[6], [11], [12].

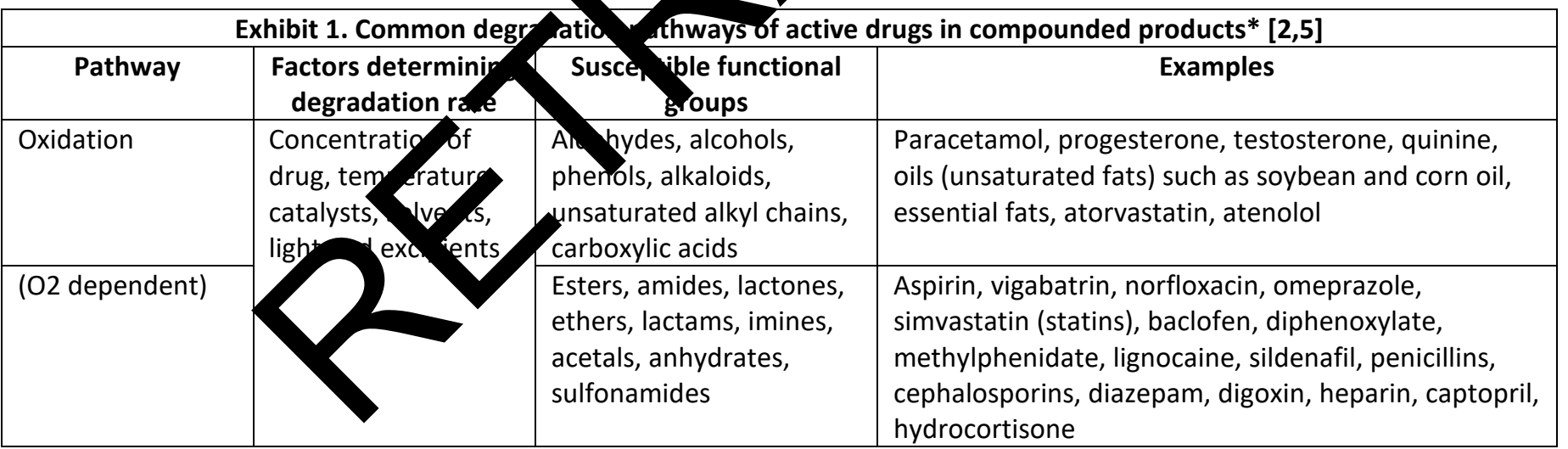

*Drug molecules with more than one functional group can be more easily degraded. In fact, many drugs contain more than one functional group, being susceptible to both oxidation and hydrolysis, e.g. atenolol (contains amide and alcohol groups)

It is essential that the active ingredient does not interact with any excipients originating from the dosage form being crushed for reformulation (e.g. tablet lubricants and fillers), or from additives in the new formulation (antioxidants, preservatives, suspending agents, colourants, emulsifiers). Interactions with other ingredients can result in physical instability of the product, such as precipitation of the active drug or phase separation ('cracking') of a cream, affecting drug solubility, absorption and bioavailability. To minimize the risk of a compounded medicine degrading, short-term expiry dates are used (e.g. 28 days for oral and topical products, or 24 hours for parenteral formulations), unless stability studies have been conducted and indicate otherwise $[2,5]$.

Potential Risks: FDA-approved drugs are made and tested in accordance with GMPs, which are federal statutes that govern the production and testing of pharmaceutical products. In contrast, compounded drugs are exempt from GMPs, and 
testing to assess product quality is inconsistent. Unlike FDA-approved drugs, pharmacy-compounded products are not clinically evaluated for safety or efficacy. The NABP further describes compounding as the result of a practitioner's prescription drug order based on the practitioner/patient/pharmacist relationship in the course of professional practice. In addition, compounded preparations do not have standard product labeling or prescribing information with instructions for safe use. Compounding pharmacies are not required to report adverse events to the FDA, which is mandatory for manufacturers of FDA-regulated medications. Some pharmacies engage in activities that extend beyond the boundaries of traditional pharmacy compounding, such as large-scale production of compounded medications without individual patient prescriptions, compounding drugs that have not been approved for use in the US, and creating copies of FDA-approved drugs. Compounding drugs in the absence of GMPs increases the potential for preparation errors.
When compounding is performed on a large scale, such errors may adversely affect many patients. Published reports of independent testing by the FDA, state agencies, and others consistently show that compounded drugs fail to meet specifications at a considerably higher rate than FDA-approved drugs. Compounded sterile preparations pose the additional risk of microbial contamination to patients. In the last 11 years, three separate meningitis outbreaks have been traced to purportedly 'sterile' steroid injections contaminated with fungus or bacteria, which were made by compounding pharmacies. US-FDA and the NICHD, have worked together to develop product labeling for pediatric patients for existing and new drug molecules coming to the market, if the drug has been or could be used in children. When compared to GMPs, the manufacturing standards a qun by FDA for commercial products, there are mult recogn deficiencies inherent in extemporaneous forma tion-com ounding suitable for pediatric patients 1,13$]$. Exhibit 2. Why Is Off-Label Use of Drugs in Childrer fill a Prob m?

- Lack of specifications required for component development by co, pou 'ing pharnacies.

- No onsite testing of active ingredients and excipients for puritynatency, co tent and stability.

- No onsite specifications or testing of product containers an closures.

- Site-to-site variations in compounding procedures, equipm ht, and th degree of product handling/manipulation.

- Lack of environmental control, which might lead to intention anination and generation of degradation products due to inconsistent exposure to light, temp raty nd processing controls.

- Lack of testing of finished products for purity-notend ontent or stability.

- Stability data for establishing expiry date $\mathrm{co}$ poun pd products are derived from published data, where preparation methods likely vary from lo fl met adcor simply default expiry periods defined by regional pharmacy regulations and "best pr

- Published preparation methods vide only portion of the information needed to consistently prepare a stable potent final product.

- Limited options available t mask bad- $t$ ting active ingredients.

- The dose administration cechno'ogies useu such as droppers, syringes, scoops, spoons, etc., vary between sites and between prescript, fil

- Weak regulatory

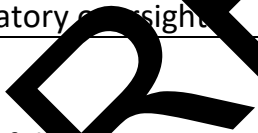

Quality Control: One of the stest growing and most important areas of pharmaceutical comp unding is that of quality control. Quality must be built-in to the preparation from the beginning steps to evaluating the final preparation. The extent of quality control applied to a product should be proportionate with the level of risk the finished product could pose to the patient and should be judged on a case by case basis. Even where a given formulation has been shown to achieve suitable physical, chemical and microbiological stability, the bioavailability and palatability of the preparation may be unproven. Consideration should be given to the individual patient's age and condition, whether the product will be ingested or used topically, as well as the potency of, and risk posed by, the active substances and/or excipients. Where it is not practical to carry out this testing (e.g. due to the batch size, urgency, etc.), other suitable methods should be implemented to ensure that the appropriate quality is achieved, in accordance with the risk assessment carried out. At a minimum, the starting materials and finished product should be examined visually before supply to a patient. There are several quality control tests that can be done within the pharmacy and others can be sent to a contract laboratory. The following quality control tests can be considered for the respective dosage forms.

- Oral and topical liquids (solutions, suspensions, emulsions): Weight/volume, $\mathrm{pH}$, specific gravity, active drug assay, globule size range, rheological properties/pourability, physical observation (color, clarity), physical stability (discoloration, foreign materials, gas formation, mold growth). 
- Hard Gelatin Capsules: Weight-overall average weight, weight individual weight variation, dissolution of capsule shell, disintegration and/or dissolution of capsule contents, active-drug assay, physical appearance (color, uniformity, extent of fill, locked), physical stability (discoloration, changes in appearance).

- Ointments, Creams and Gels: Theoretical weight compared to actual weight, $\mathrm{pH}$, specific gravity, active drug assay, physical observations (color, clarity, texture-surface, texture-spatula spread, appearance, feel) and rheological properties.

- Suppositories, Troches, Lollipops and Sticks: Weight, specific gravity, active drug assay, physical observation (color, clarity, texture of surface, appearance, feel), melting test, dissolution test, physical stability.

- Parenteral preparations: Weight/volume, physical observation, $\mathrm{pH}$, specific gravity, osmolality, assay, color, clarity, particulate matter, sterility, pyrogenicity [5,6], [14].

Compounding Support: Numerous agencies, companies, organizations, etc., are available to assist pharmacists in compounding. Information, chemicals, supplies, and equipment are readily available. Chemical and supply companies have increased in size and number in recent years and many provide information on compounding, incompatibilities, and stability. Specialty compounding organizations have developed over recent years and genera' provide full-line services and products to the compoundin pharmacist. Many national organizations provide continuing professional education programs in both non-sterile nds rile compounding. These entities provide services to mpoy ding pharmacists ranging from selling only comp and aids to providing only chemicals. Others offer ad ronal sel res to include formulas as well as consulting 6,pert by teleprone or via the internet. This service car assist in th process of compounding a particular product nat may be difficalt.

in the context of ex
- Extemporaneously pree
formulated and prepared in compliance with current
legal requirements and standards.
Preparation and quality control arrangements are to be
documented and in compliance with current GPP
requirements.
- All products prepared should be quality suitable for
their intended use
Products are released for patient use only by a
pharmacist.
Documentation and records should comply
recommendations in this guidance document.

Training and Experience: Pharmacists involved in upgrading and increasing the traditional aspects of extemporaneous compounding need to keep current with all the new tools of their trade, retrieve the old from storage, and put in a bit of practice using their scientific background and their art before they will be comfortable in exhibiting their skills. The pharmacist must ensure that the staff, facilities and systems in place are capable on a day-to-day basis of providing an adequate quality service able to meet the needs of patients. There should be an adequate number of competent personnel at all times. All staff involved in extemporaneous preparation must receive training Appropriate to their role. Typically, this will: (a) provide them with knowledge in good extemporaneous preparation practice, local practices including health and safety, formulation, expin as and quality assurance appropriate to the level involvem nt, assessment of risk and medication error potent the ph rmacy, its products and services provide (b) de onst ate competency in the necessary e empg heous preparation skills and pharmaceut al ations d dilutions. During training, staff must be refully so ery ed and checked and trainees must under and the lims of their responsibilities. When concidering pro iding additional services of compounding in an stitution, pharmacists should not expect that this will change great eal of their practice in time consumed for umpound $\mathrm{ig}$. A written training program should be available an metion of training should be documented. This applies all personnel working in the preparation area, including those not directly involved in preparation processes (e.g. cleaning staff). [14].

Equipment: The equipment needed will be determined by the type and extent of the services one chooses to provide. Many pharmacies already have clean air environments (e.g., laminar air flow hoods, isolation barrier systems) where aseptic compounding of sterile solutions is performed. These same units can be used to compound other sterile preparations such as eye drops. A balance, preferably electronic, is essential. Ointment slabs (i.e., pill tiles), along with spatulas of different types and materials, should be purchased. A few mortars and pestles (i.e., glass, ceramic, plastic) and some glassware should be secured. A policy for the segregation of specific equipment for certain product types, including cytotoxic drugs and external products, should be available; this should take into account local COSHH assessments. Equipment should be calibrated and validated to demonstrate satisfactory performance. Measures should be calibrated for specific volumes and should have levelling lines. Glassware should be inspected regularly, before and after each use, for chips and cracks, and replaced as necessary. Consideration should be given to the merits of other materials. Stainless steel of a suitable grade is a good material but care must be taken with some agents as it is susceptible to oxidation. Plastics are susceptible to surface scratches and stains. The accuracy of balances should be checked on a regular basis and records of this process maintained. It may not be necessary to buy a roomful of equipment, but one should 
purchase what is needed to start the service and should build on it as the service grows and expands to different arenas. Much of the equipment used today in compounding has changed. Today, electronic balances are used more often than torsion balances; micro-pipets are commonplace; and ultrafreezers are sometimes required in addition to standard refrigerator freezers. This area is constantly changing and the compounding pharmacist should be aware of the available technology to prepare accurate and effective prescriptions. Becoming acquainted with the local representative for a laboratory supply company is helpful [2], [6], [14-19].

Environment: A separate area for traditional compounding is recommended, rather than simply cleaning off a small area of the dispensing counter. The compounding pharmacist needs a clean, neat, well-lit and quiet working area. If aseptic compounding is considered, a clean air environment (e.g., laminar air flow hood, isolation barrier system) should be used. The actual facility to be used depends on the level and volume of compounding to be done. Extemporaneous compounding takes place in community and hospital pharmacies. There are usually specialist compounding pharmacies in major towns and cities, but any pharmacy may undertake compounding as long as they have appropriate facilities according to state-based legislation (e.g. allocated clean bench, specific compounding equipment). There should be a dedicated facility/room for extemporaneous preparation. However, for pharmaci preparing small quantities of a limited range of products fo immediate dispensing then a designated area or temararily designated area is permissible. The area must c c prly demarcated during preparation. Measures mus be tak o reduce any risk of cross- contamination. $9 /$ ov roduct should be handled at a time. Premises and quipmen hould be easy to clean. Following maintenan or vair, tholough cleaning and, where appropriate, sinfection bould take place. Environmental condition, including termperature, humidity and lighting, must be equ to nermit safe and comfortable preparation gredic pror to limit the degradation of ingredien Faci les sh laid out in a way to provide adequate sß for fortivities undertaken. Work should flow logically betw working areas without the risk of product cross- contaminan. Complex compounding is performed in a pressurized clean room using a laminar flow cabinet, cytotoxic drug safety cabinet or an isolator. Many public and private hospitals maintain large aseptic compounding facilities to provide individualized dosing or commercially unavailable formulations. Areas and equipment should be cleaned effectively with a suitable detergent before and after use. Equipment should be rinsed with an appropriate grade of water after washing (potable or sterile water for irrigation/injection) and then dried. Critical surfaces should be effectively sanitized with a suitable agent such as $70 \%$ alcohol before use. Sinks should be available for washing up of equipment, with separate facilities for handwashing. Sinks should be a suitable distance away from the preparation areas to reduce the risk of microbial contamination from water splashes. Washing and cleaning activities should not themselves be a source of contamination. All equipment should be visibly clean prior to use. Ensure that all residues of cleaning agent have been removed Containers and lids should be checked to ensure that they are clean and dry before use. Adequate pest control measures should be taken. [6], [10], [19]

Formulas: Consistency of the compounded product is important. Formulas should be developed or obtained and tried to assure that each time an extemporaneous product is prepared, the methods used, ingredients added, and the order of steps is documented. This accomplishes three things. First, it provides the methodology for each person involved or requested to provide such service the information necessary to do so properly. Second, it ovn consistency from batch to batch. Third, if the prod does no urn out the way expected, a stepwise methodghgy ists for $r$ viewing and determining what happened ar if revisio an mprovements are needed [6], [15].

Chemical and Sup es. one is going to prepare a topical produ , a hicle (e.g. cream, ointment, gel) and the active ingrodients (e.s. either finely ground product from an available alet or injectiolor pharmaceutical-grade chemicals) would be require. One needs proper dispensing containers for the edicatio In short, a relationship with providers that carry cho and supplies is important. Pharmacists have been xing chemicals and other materials for prescription compounding throughout history. In the past, these chemicals and materials have been obtained from natural products, raw materials, and household ingredients. Today, compounding pharmacists use chemicals from various reliable commercial sources, depending on their availability [9], [15-18].

Documentation: These give processing, packaging and release instructions. For products regularly prepared, master documents which combine the specification and product specific instructions should be produced and independently checked. Alternatively, a simple final product specification may be produced in addition to the master document. Master documents should be clear and detailed and should have a standardized style within any one pharmacy. Simple stepwise instructions for preparation, along with any specific notes or cautions (e.g. COSHH requirements) should be included on the master document for each product. A record should be kept of the key stages of processing, packaging and release of products to provide an audit trail of the quality relevant facts of the history of an extemporaneous product during preparation. This is normally achieved by completion of an individual worksheet, usually by photocopying the master document. Worksheets will vary for each pharmacy but should include: the name and formula of the product, and the source of the formula; a unique identification number to enable traceability; the manufacturer, batch numbers of each starting material (or QC reference number); the date of preparation etc. Labels must comply with all statutory and professional requirements, and should include 
the following information: the name of the product, and where necessary the nature of the salt and any waters of crystallization; quantity and strength of active ingredients; the pharmaceutical form etc. [6], [20].

\section{Types of Compounding}

Active pharmaceutical ingredients can be incorporated into a wide array of products including creams, eye drops, nasal sprays, oral dosage forms or intravenous infusions. In Australia, products may be classified into simple or complex compounding (Table 1). Simple compounding can be performed by any pharmacist and is a core competency of pharmacy training. Complex compounding requires additional training and evidence, as described by the Pharmacy Board of Australia's guidelines on compounding. Finland, Italy, and Scotland prepared mainly powder, while Belgium, Croatia, France, and Switzerland prepared mainly capsules. Practice in Germany, Spain, and Slovenia involved preparation of a less well-defined combination of liquid, powders, and capsules.

Table 1. Classification of simple versus complex compounding [5]

\begin{tabular}{|c|c|c|}
\hline Type & Explanation & Examples \\
\hline Simple & $\begin{array}{l}\text { All pharmacists have training during their } \\
\text { undergraduate degree to prepare these } \\
\text { products. } \\
\text { Involves well-established preparations } \\
\text { published in reputable literature, e.g. the } \\
\text { Australian Pharmaceutical Formulary and } \\
\text { Handbook, or formulae for which some data } \\
\text { are available regarding quality, stability, } \\
\text { safety, efficacy and rational design. }\end{array}$ & 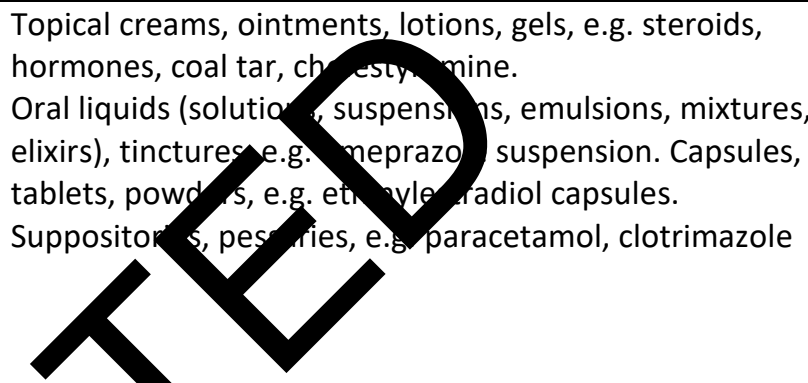 \\
\hline Complex & $\begin{array}{l}\text { Pharmacists require further postgraduate } \\
\text { training in association with self-assessmen } \\
\text { of relevant competencies and } \\
\text { documentation of the specific } \\
\text { competencies in a continuing pro } \\
\text { development plan. Specialized faci } \\
\text { (sterile room with positive } \\
\text { equipment (laminar flow solat dry } \\
\text { sterilization oven) are so res }\end{array}$ & $\begin{array}{l}\text { safenteras. morphine, clonidine. Ingredients with a } \\
\text { safety hazard, e.g. cytotoxics, hormones } \\
\text { Single nit micro-dose (<25 mg of drug or no more than } \\
25 \% \text { y of a dosage form), e.g. naltrexone. Modified- } \\
\text { progesterone capsules } \\
\text { Ophthalmic preparations, e.g. phenylephrine, } \\
\text { tropicamide, ciprofloxacin }\end{array}$ \\
\hline
\end{tabular}

Ambulatory-Care Compounding: If individ" 's can wa they are considered mobile or ambulatory (i.e.) they are not bedridden). Consequently, most pl macists an involved in ambulatory care, and most ambul cory patients are outpatients. Actually, the term 1 be anplied to homecare patients and even iztiol ized atients who are mobile. One general ch acter tic of oulatory patients is that they are generall, resp obtaining their own medication, storing it, pres, ing it (if necessary), and taking it. It seems almost incongruous bat in health care today as we become more aware that patients are individuals, respond as individuals, and must be treated as individuals that some health-care providers appear to be grouping patients into categories. They are grouped in categories for treatment, for reimbursement from a third party, or for determining levels of care in managed-care organizations and using fixed-dose products provided by pharmaceutical manufacturers that are available because the marketing demand is sufficiently high to justify their manufacture and production. Why should the availability or the lack of availability of a specific economically profitable commercially available product dictate the therapy of a patient? Pharmacists have an opportunity to extend their activities in patient care as the emphasis continues to shift from inpatient care to ambulatory care. Ambulatory care, however, is so diverse and involves so many disciplines that sometimes it is difficult to understand it; and, it changes rapidly. Also, ambulatory care could generally encourage a team approach to health improvement, prevention, health maintenance, risk assessment, early detection, management, curative therapy, and rehabilitation. Ambulatory care offers various opportunities for individualizing patient care through pharmaceutical compounding. In fact, it is the area where most compounding pharmacists' practice. Pharmacists' roles in ambulatory care patients can include, among others

- Dispensing

- Compounding

- Counseling

- Minimizing medication errors

- Compliance enhancement

- Therapeutic drug monitoring

- Minimizing expenditures

Most reimbursement for ambulatory patients comes from the dispensing or the compounding process. Little financial consideration is given to counseling, minimizing medication errors, compliance enhancement and therapeutic monitoring. However, these activities are important and should be 
performed. Because of the unique nature of compounded medications, counseling is an absolute must for these patients. From the above discussion of the activities of ambulatory care pharmacists, it should be evident that extemporaneous compounding can be vitally important in ambulatory patient care [6], [18-21]

Hospital Pharmacy Compounding: The ever-present responsibility of the health-care industry is to provide the best available care for the patient, using the best means to do so, and providing that care in a conducive environment. This must be sufficiently economical to not put the institution in jeopardy of being unable to continue to provide the services to the community they serve. This requires cooperation on the part of the hospital administration, the medical staff, and the employees (nurses and pharmacists in particular as regards to medication usage) and must involve the patient. One of the effective means by which hospitals, and therefore hospital pharmacies, can meet these challenges is to consider expanding extemporaneous compounding services within the hospital pharmacy. Pharmaceutical care and pharmaceutical compounding can provide cost savings to the hospital while providing needed options to the physician through problemsolving approaches and stimulating the hospital pharmacist through new challenges that allow the expression of both their skills and their art [15], [22,23]

Veterinary Compounding: The first symposium on veterinar compounding was a significant forum for discussion by and was a pivotal point in the history of eten ary compounding, occurring in September 1993. the geetip was important because it assembled an impressive, coun experts on veterinary compounding, who then set ut explal, $\mathrm{g}$ and defining the roles of the veterinarian an he prmacist 24].
Nuclear Pharmacy Compounding: Nuclear pharmacy is a specialty practice of pharmacy that has been defined as a patient-oriented service that embodies the scientific knowledge and professional judgment required for improving and promoting health through assurance of the safe and efficacious use of radioactive drugs for diagnosis and therapy. Radioactive drugs, commonly referred to as radiopharmaceuticals, are a special class of drugs that are regulated by the FDA. They are unique in that they contain an unstable nuclide (radioactive nuclide) as a part of the compound designed to localize in an organ or tissue. Since radiopharmaceuticals are radioactive, the Nuclear Regulatory Commission or a similar state agency is involved in regulatory matters relevant to radiop nuticals [25-27].

Risk Management Legal Background harmacs my comply with all legislation relevant to the ractic of phar acy in the jurisdiction where

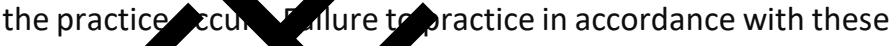
requirem ts may ive to action by one or more respo mole thorities. hese matters may then be refered to the Roard for a vopriate action under the National Law or law a co-regulatoly jurisdiction. Non-compliance with these suidelines nd the practice standards and guidelines relevant compor ding may also be notified directly to the Board for ap arulatory jurisdiction. Non-compliance may be reported by an indıvidual lodging a notification form, or through other means such as notification of outcomes of audits carried out by a state/territory pharmacy premises regulatory authority or responsible body [5].

\begin{tabular}{|c|c|}
\hline $\begin{array}{l}\text { Acetaminophen, codeine mosp te, an affeine capsules and tablets } \\
\text { Amobarbital sodium cal } \\
\text { Amyl nitrate inhalant } \\
\text { Chloral hydrate capsules, syro, and suppositories } \\
\text { Codeine phosphate injection, oral solution, and tablets } \\
\text { Codeine sulfate tablets } \\
\text { Colchicine injection and tablets } \\
\text { Digitoxin tablets } \\
\text { Digoxin elixir and tablets } \\
\text { Ephedrine sulfate capsules and injection } \\
\text { Ergonovine maleate injection and tablets } \\
\text { Ergotamine tartrate tablets } \\
\text { Hydrocodone bitartrate tablets } \\
\text { Hydrocodone bitartrate, aspirin, and caffeine tablets } \\
\text { Hydromorphone hydrochloride suppositories } \\
\text { Levothyroxine sodium for injection }\end{array}$ & $\begin{array}{l}\text { Morphine sulfate oral solution and tablets } \\
\text { Nitroglycerin sublingual tablets } \\
\text { Opium tincture } \\
\text { Oxycodone tablets } \\
\text { Oxycodone hydrochloride oral solution } \\
\text { Paregoric } \\
\text { Phenazopyridine hydrochloride tablets } \\
\text { Phenobarbital capsules, elixir, and tablets } \\
\text { Phenobarbital sodium injection } \\
\text { Pilocarpine hydrochloride ophthalmic solution } \\
\text { Potassium bicarbonate effervescent tablets for ora } \\
\text { solution } \\
\text { Potassium chloride oral solution } \\
\text { Potassium gluconate elixir and tablets } \\
\text { Potassium iodide oral solution } \\
\text { Salsalate capsules } \\
\text { Sodium fluoride oral solution and tablets } \\
\text { Thyroid tablets }\end{array}$ \\
\hline
\end{tabular}




\section{Alternatives to extemporaneous preparation}

- Therapeutic substitution: The use of a licensed medicine from the same therapeutic classification should be considered and may provide a better clinical option than the use of an extemporaneously prepared medicine which has limited data to support its formulation and stability. The use of a less potent steroid rather than diluting a potent agent is an example where a therapeutic alternative may eliminate the need for an extemporaneous preparation. There are great opportunities for oncology pharmacists, as well as community pharmacists, as a resource for educating and monitoring patients receiving oral chemotherapy to ensure dosing accuracy, safe administration, and proper disposal of hazardous drugs. Oncology pharmacists face a constant challenge with patients who cannot swallow oral anticancer drugs, making extemporaneous oral liquid preparation a requirement. Improper extemporaneous preparation of these agents, especially with the traditional chemotherapy with a narrow therapeutic index, may increase the risk of over- or underdosing [28].

- Use of an imported product: The preparation selected should be licensed for use in a country with equivalent or similar licensing arrangements and regulatory standards to the UK (e.g. EU, Canada, Australia). This will provide the requesting pharmacist with assurance that the quality, safety and efficacy of the medicine have been reviewed a competent regulatory authority. Although clinicians d not doubt the efficacy and utility of captopril in managing children with heart failure, concerns exist abo op mal dosing schedules and toxicity. This formulat is lic cad in the Bristol-Myers Squibb, Australi- bu. as not available in the UK [29].

- Use of a 'Special' manufactured in MH licenseananit: The benefit of purchasing a 'Sp clal' is that he product should be made to a validat a formula with supporting stability data in accordance vith principles of GMP. Licensed 'Specials' unitare ularly ispected by the MHRA to ensure the prin ples an cheld. However, the purchasing pharn cist need to review the supporting document on (e.g. specification, Certificate of Analysis/Conformity, b _TSE statement) to assess whether the product is of appropriate quality. Information on Specials manufacturers is available in the BNF. Specials may be used to meet a variety of patient needs, for example, for babies, children and older people, and in specific areas such as dermatology and palliative care [30].

- Use of soluble or dispersible tablets: This practice presents fewer health and safety risks than crushing tablets, which can expose the carer to potentially harmful dusts via inhalation. When dispersing tablets, the dose should be prepared and administered immediately, as stability cannot be guaranteed. It should be noted that slow or modified release preparations should not be used in this manner. If the active is not soluble, it can lead to inaccuracy of dosing through a lack of dose uniformity and reproducibility. This is a major consideration when no suspending agents are used, especially when the person administering the dose is inexperienced and the dose is small [31]. Milk has been explored as a vehicle in liquid formulations showing potential for solubilizing drugs while maintaining the stability of the emulsified vehicle [32].

- Cutting tablets: Tablets cannot be cut with great accuracy of dose and research suggests that the variability may range from $50 \%$ to $150 \%$ of the desired dose even when using commercially available tablet cutters.

- Use of a preparation intended for a different route: The use of a suitable preparation intended for a different route of administration can sometimes be a practical alternative; for example, the use of an injection solution orally, or an oral solution rectally. own this practice has its own inherent risks and pharma should ensure that the presentation used whe absor ed by this route and that it will be toler ed by th ratir. When using an injection by the or route Considertion should be given to the possibily or absor cion and elevated peak levels, the pote al for ra, d degradation due to exposure to garic id and pholems with first-pass metabolism. The nH of an in ction should also be considered, as extremes of $\mathrm{pH}$ can adversely affect the gastric mucosa [20].

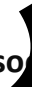

ated with extemporaneous preparation lation failure: An insoluble drug suspended in a suitable vehicle may be less susceptible to drug degradation, but may settle out of the suspension over time, leading to sedimentation and caking. In this state, there will be a higher concentration of drug at the bottom of the bottle than at the top. In 2006, the FDA conducted a limited survey of compounded drugs. Of 36 samples tested by the FDA, 12 failed at least one quality test, for a failure rate of $33 \%$. Further, oral hormone dosage forms containing multiple active ingredients showed poor content uniformity, with random variation in all three active ingredients from capsule to capsule [8], [20].

- Microbial contamination: The choice of preservative for a formulation needs to take into account a number of factors including $\mathrm{pH}$, physical compatibility and the intended patient group. Unpreserved preparations should be stored in a refrigerator and assigned a short shelf-life to limit microbial growth. A maximum shelf-life of 7 days at $2-8^{\circ} \mathrm{C}$ should be assigned to unpreserved oral liquid preparations unless sufficient validation work has been carried out to support an extended shelf-life. Essential macronutrients and micronutrients infused via PN solutions are an essential part of the care of infants and children who cannot or should not be fed enterally. Infants and children require a greater amount of fat in their diet than do adults to support growth and development. The use of IVFE provides a concentrated source of calories and prevents the development of essential fatty acid deficiency in neonates fed exclusively via the parenteral route. The AAP recommends IVFE doses of up to $3 \mathrm{~g} / \mathrm{kg} /$ day 
(approximately $30 \mathrm{kcal} / \mathrm{kg} /$ day) for use in infants. A variety of microorganisms have been found in residual IVFE after completion of the infusion [33]. Microorganisms could potentially be introduced during reformulation of nonsterile products. For example, if Candida albicans is inadvertently introduced into freshly prepared multi-dose citric acid solutions that are to be used orally for cough reflex testing, immunocompromised individuals could become infected. Regular monitoring of the environment, equipment and procedures is essential to ensure quality and sterility is maintained. The consequences of failure can be catastrophic, for example methylprednisolone injections from a single compounding pharmacy in the USA resulted in 137 cases of Aspergillus fumigatus meningitis and 12 deaths [2].

- Calculation errors: Common calculation errors associated with extemporaneous preparation include errors when converting units from one to another (e.g. milligrams to micrograms, conversions from weight in volume to millimoles). Dangers of potent ingredients and calculation errors, particularly where the strength of one or more ingredient is stated in a historical or non-standard fashion. Similar reports have originated from the US, with the death of a child from a super-potent imipramine liquid, and a fiveyear-old child who received a 1,000-fold overdose of clonidine. Problems can also arise when doses can be prescribed as free base or salt, leading to potent calculation errors when making and administerin preparations (e.g. two-fold errors if caffeine confused with caffeine base). Some of these been attributed to inconsistent labelling a example, strengths expressed per millire er $5 \mathrm{mr}$ spoonful. Clinical pharmacists in pediatr and neonatology help in dose calculat on an dosage rorm modification. The increased n for calc ations and dilutions of pediatric medic es, and a requirement to adjust dose of an indivic 1 crent based on age, gestational age, weight sun area orovide a greater opportunity for a clinic pha ast in medication management pross. help to prepare pediatric/neonatal fol dary [34], [41].

- Starting materials: Care ould also be taken with the use of cariogenic sugars (e.g. sucrose) in pediatric formulations as it has been associated with dental cavities. It is therefore important to list all such excipients on the product label so that end-users are made aware of their presence in the formulation. In a 2004 published analysis sponsored by STD Pharmaceuticals, all samples purchased from three compounding pharmacies failed content testing for a $3 \%$ sodium tetradecyl sulfate solution for injection (range 2.59-3.39). Significant concentrations of the contaminant carbitol were found to be present in samples from all three sources (0.33-4.18), suggesting possible use of a nonpharmaceutical grade chemical [35].
- Patient acceptability issues: Consideration should be given to the palatability and presentation of oral liquid medicines as there is a good argument that taste is crucial to achieving good compliance in children, especially for the treatment of longstanding conditions such as in cardiology. Extemporaneously prepared oral drug formulations can also be plagued by poor palatability characteristics which can compromise patient adherence. This lack of childfriendly formulations affects $40 \%$ of the global population, subjecting pediatric patients to avoidable adverse drug events, reduced compliance with medication regimens, limited access to new medications, and prolonged treatable illnesses. The pressing need for child-friendly, palatable medications suitable for administration to both infants and young ch as been stressed (through regulatory guidan by both the European Medicines Agency and the US PA. Pred isone is a bitter-tasting corticosteroig used it anti-inflammatory and immunosu ressa effecu to treat a wide variety of conditis s th ads and children. For pediatric patie s, an al vo form of administration can be p duc by crushng solid oral prednisone formulations in arder to oid swallowing difficulties and/or achieve weight-basea dosing. This approach, however, creates extre ply poor taste and mouth feel properties which are object pnable to pediatric patients. While these limitations somewhat mitigated by the use of proprietary oral liquid formulations of prednisone, such products still retain their profoundly bitter taste characteristics. It is estimated that approximately half of children refuse to take even a liquid form of prednisone, with the large majority of those reporting bad taste as the single major reason for noncompliance. Efforts to mask flavors using sweetening agents, coatings, agglomeration, or microencapsulation often result in poorly-controlled, heterogeneous particle size distributions that result in a gritty or granular mouth feel and can provide ineffective taste masking, characteristics that may also compromise patient acceptance. The design of an ideal paediatric formulation needs to consider the following factors: (i) producing minimal impact on the lifestyle of the child, manifesting as the lowest dosage frequency and a palatable product, (ii) provision of individualized dosing or dose banding appropriate for effective therapy, (iii) sufficient bioavailability, (iv) non-toxic excipients in the formulation, (v) convenient and reliable administration and (vi) robust production process at minimal cost [20], [36-39].

- Health and safety risks: When handling hazardous products, units should be equipped with suitable containment devices and systems should be put in place to eliminate the risk of cross-contamination.

- Therapeutic risks and clinical consequences: Any inaccuracy of dosing associated with medicines that have a narrow therapeutic index can lead to significant morbidity, whether due to under-dosing leading to treatment failure or overdosing leading to toxicity. By contrast, any 
inaccuracy of dosing associated with drugs with a wide therapeutic index may have little or no impact on the therapy.

- Associated clinical risk factors: The majority of patients receiving extemporaneously prepared products, in particular oral liquid medicines, tend to be from vulnerable patient groups (e.g. neonates, children, stroke victims) who are either unaware of ill-effects associated with their treatment or who cannot communicate with their clinician.
Coupled with this, extemporaneous preparations may not be routinely identified as high-risk therapies by pharmacists and therefore such treatments are not commonly given the level of scrutiny and close monitoring they require. Therefore, when embarking on the use of an extemporaneously prepared product, the pharmacist should ensure that systems are in place to monitor the effectiveness of the therapy [20].

\footnotetext{
Exhibit 5. Extemporaneous preparation or compounding standards as set out in the Medicines [44]

- A product is extemporaneously prepared only when there is no product with a marketing authorization available and where you are able to prepare the product in compliance with accepted standards.

- Staff involved are competent to undertake the tasks to be performed.

- The requisite facilities and equipment are available. Equipment must be maintaingd in od orde to ensure that performance is unimpaired, and must be fit for the intended purpose.

- The professional associate (Pharmacist) is satisfied as to the safety and appr riate ss of th irormula of the product.

- Ingredients are sourced from recognized pharmaceutical manufacturers $d$ at a qual accepted for use in the preparation and manufacture of pharmaceutical products. Where apn oriate, re van fegislation must be complied with.

- Particular attention and care are paid to substances which may bnazardou nd require special handling techniques.

- The product is labelled with the necessary particulars, includj g an expiry date and any special requirements for the safe handling or storage of the product.

- If you are undertaking large-scale preparation of medicinal p ducts, al elevant standards and guidance are adhered to.

- Records are kept for a minimum of 2 years. The reco $\$ \mathrm{mb}$. their source; the batch number; the expiry data-wher preparation is dispensed in response to a prescription, the patient's and prescription details and the $d^{\prime}$ (o) ispen ng; the personnel involved, including the identity of the pharmacist taking overall responsibility
}

\section{Risk Management}

The European Pharmacopoeia Preparations specifies the neces particular dosage forms, as form monographs, wh extemporaneously prê, re testing in the European rmacopeia monograph deal with the appearance of produc identity and purity tests, uniformity and reference standards. The extent of quality control applied to a product should be proportionate with the level of risk the finished product could pose to the patient and

\section{lograp on Pharmaceutical} be applied to are so ap able in the case of The requirements on

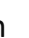
(1)

(n)

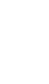

should be judged on a case-by-case basis. Consideration should be given to the individual patient's age and condition, whether the product will be ingested or used topically, as well as the potency of, and risk posed by, the active substances and/or excipients. Where it is not practical to carry out this testing (e.g. due to the batch size, urgency, etc.), other suitable methods should be implemented to ensure that the appropriate quality is achieved, in accordance with the risk assessment carried out. At a minimum, the starting materials and finished product should be examined visually before supply to a patient [40]. 


\begin{tabular}{|l|l|}
\hline \multicolumn{1}{|c|}{ Exhibit 6. Reference texts and other sources of information relevant to compounding } \\
\hline - & Martindale: The Complete Drug Reference \\
- & Intersel's Stability of Compounded Formulations - Trissel LA \\
- & Australian Don't Rush to Crush Handbook - The Society of Hospital Pharmacists of Australia \\
- & Phandbook on Injectable Drugs - American Society of Health - System Pharmacists \\
- The Art, Science and Technology of Pharmaceutical Compounding - Loyd Allen \\
- Australian Injectable Drugs Handbook - The Society of Hospital Pharmacists of Australia \\
- $\quad$ Remingl's Pharmaceutical Dosage Forms and Drug Delivery Systems - Loyd Allen \\
- Guide to Good Manufacturing Practice for Medicinal Products (can be accessed from the TGA website) \\
- Guidelines for the Safe Prescribing, Dispensing and Administration of Cancer Chemotherapy (can be accessed from \\
the Clinical Oncological Society of Australia website)
\end{tabular}

the Clinical Oncological Society of Australia website)

\section{Role of the Pharmacists}

Compounding preparations extemporaneously on lawful prescription for individual patients is within the pharmacist's purview. Pharmacists and pharmacies are licensed and inspected by the states; if a state inspection uncovers "manufacturing under the guise of compounding," it refers the matter to the FDA, which can apply cGMP expectations to the pharmacist/pharmacy. When a pharmacist engages in largescale production of preparations without prescription or evidence of pending refills, trending, etc, the practice can br regarded as manufacturing; cGMP compliance will be expected including an approved NDA or ANDA. Today, however, compounding pharmacies have begun to flourish and ance again becoming a vital component of pharmacy pr ctice. any health care professionals recognize that, fon so pecim patient populations, there is a growing $y=d$ to a pound certain medications because they are ilable thugh conventional manufacturing method Wherea the services provided by compounding pharp cies give ma patients options with regard to drug ther $*$ con ounding pharmacists must be properly trained to so rize in this aspect of pharmacy practice and $n$ sth ve thap opriate resources and references to com and ality meurcations. Pharmacists are responsible for ensun at extemporaneous preparations are compounded according to compounding guidelines and standards with respect to purty, quality, stability, packing, record keeping, and other appropriate pharmacy practices. Compounding pharmacists must work closely with the prescribing physician to ensure that patient goals are met. A positive therapeutic outcome for the patient is the fundamental goal for all health care professionals, and, with that goal in mind, the services provided by compounding pharmacies can continue to make pharmacists an indispensable part of the health care team $[42,43]$.

\section{Conclusion}

Pharmacy comp anding prov pharmacists with a unique opportunity to their time-honored profession. It is becoming a even re in ortant part of pharmacy practice in the fut $\Leftrightarrow$ cluding $t$ involved in community and hospital care, nursing re, home care, veterinary and other specialty actues. It is a ractice where the clinical expertise can be nerged with the scientific expertise of pharmacists to make a isible pha naceutical care. Pharmacists are to be encouraged 1. in ompounding but should be aware of the core of formulating a specific drug product for a specific patient. This is Imr.ortant in providing pharmaceutical care.

\section{Article Summary}

It is important to use a pharmacist's expertise to adjust dosage quantities, frequencies, and even dosage forms for enhanced compliance. All pharmacists should understand the options presented by compounding. Pharmaceutical compounding is increasing for a number of reasons, including the availability of a limited number of dosage forms for most drugs, a limited number of strengths of most drugs, home health care, hospice, the non-availability of drug products/combinations, discontinued drugs, drug shortages, orphan drugs, new therapeutic approaches and special patient populations. Extemporaneously compounded medicines may be useful when a required dose or dose form is unavailable commercially, or for individualized dosing. There are numerous established compounding formulae available, and new formulae may be developed with the help of formulation guidelines and professional advice. Unlike registered medicines, compounded preparations have not generally been assessed for safety and efficacy. Their use is off label and is based on extrapolation from the component ingredients. Short-term expiry dates are provided for compounded products unless their stability has been assessed. 
Abbreviations: Good Compounding Practices (GCPs); Joint Commission on Accreditation of Health Care Organizations (JCAHO); National Association of Boards of Pharmacy (NABP); Control of Substances Hazardous to Health (COSHH); Extemporaneous preparations (EPs); National Institute of Child Health and Human Development (NICHD); Medicines and Healthcare products Regulatory Agency (MHRA); Transmissible Spongiform Encephalopathy (TSE); Bovine Spongiform Encephalopathy (BSE); Intravenous Fat Emulsion (IVFE); American Academy of Pediatrics (AAP); National Association of Boards of Pharmacy (NABP); New Drug Application (NDA); Abbreviated New Drug Application (ANDA).

\section{References}

1. Taylor S, Hays C, Glass B. Compounding medications in a rural setting: an interprofessional perspective. J Multidiscip Healthc. 2018;11:197-204. Published 2018 Apr 20. doi:10.2147/JMDH.S156161

2. Falconer JR, Steadman KJ. Extemporaneously compounded medicines. Aust Prescr. 2017;40(1):5-8.

3. Gullapalli RP, Mazzitelli CL, Charriez CM, Carpenter DJ, Crean RD, Carter B, Perera P. Extemporaneous preparation strategy for early phase clinical studies. Int J Pharm. 2018 Oct 5;549(1-2):150-160. doi: 10.1016/j.ijpharm.2018.07.059. Epub 2018 Jul 25. PubMed PMID: 30055303.

4. Brown G. The Value of Drug Stability Studies and Their Publication. Can J Hosp Pharm. 2018;71(2)·161162.

5. Pharmacy Board of Australia. Codes, gui policies. Guidelines on compounding 2015. http://www.pharmacyboa Guidelines.aspx

6. Allen VL. Chapter 105. Extep Poraneous escription Compounding. In: Remin on: Tho Science ond Practice of Pharmacy by Yvid. Troy, Paul Beringer,

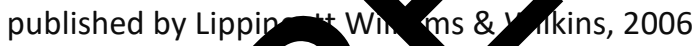

7. Costello, Ian; Lo , Pay Won o Chi Kei; Tuleu, Catherine; Yea. Vip Das ratric Drug Handling. London: Pharmao IIcal Press; 2007

8. Sellers S, Utian WH. harmacy compounding primer for physicians: prescriber beware. Drugs. 2012;72(16):2043-50.

9. Loyd VA. The Art, Science and Technology of Pharmaceutical Compounding Fifth Edition APhA Wshington, D.C.

10. Ivana Jaksic, Milica Lukic, Andjelija Malenovic, Stephan Reichl, Christine Hoffmann, Christel MüllerGoymann, Rolf Daniels and Snezana Savic, Compounding of a topical drug with prospective natural surfactant-stabilized pharmaceutical bases: Physicochemical and in vitro/in vivo characterization - A ketoprofen case study, European Journal of Pharmaceutics and Biopharmaceutics, 80, 1, (164), (2012).
11. Gudeman J, Jozwiakowski M, Chollet J, Randell M. Potential risks of pharmacy compounding. Drugs $R \mathrm{D}$. 2013;13(1):1-8.

12. Alarie H, Friciu MM, Leclair G. Stability of Dapsone in Extemporaneously Compounded Oral Suspensions. Can J Hosp Pharm. 2018;71(2):152-154.

13. Bhatt-Mehta V, MacArthur RB, Löbenberg R, Cies JJ, Cernak I, li RHP. An Algorithm to Identify Compounded Non-Sterile Products that Can Be Formulated on a Commercial Scale or Imported to Promote Safer Medication Use in Children. Pharmacy (Basel). 2015;3(4):284-294. Published 2015 Nov 11. doi:10.3390/pharmacy3040284

14. Handbook of Extemporaneous Preparation: A Guide to Pharmaceutical om unding Rowe, Lesley (Reviewed by) rited by M k Jackson, Published by the Pharmareut Press, $L$ hdon, Chicago. Australia harmac, Vol ne 29 Issue 10 (Oct 2010)

15. Allen Chap 1. Gu, elines for Compounding Pra cices Loyd Allen. The Art, Science, and chnologh $\mathrm{fPb}$ maceutical Compounding, 5th Ed n, publis, er American Pharmacists Association 2017

16. Extemporaneous formulations reference guide The P rrmaceutical Journal24 JUN 2016 By Laurence A. daberg ledicines, Ethics, and Practice: A Guide for Pharmacists, Volume 29 by Royal Pharmaceutical Society of Great Britain, 2005

18. McHugh MB. Chapter 15. Non-sterile compounding and Re-packaging. In: Mary B. McHugh. Workbook for the Manual for Pharmacy Technicians, published by ASHP, Jan 1, 2013

19. USP DI., Volume 1, Parts 1-2; Volumes 2-3 by United States Pharmacopeial Convention, 2006

20. Handbook of Extemporaneous Preparation: A Guide to Pharmaceutical Compounding by Mark Jackson, Andrew Lowey, published by Pharmaceutical Press, 2010

21. The Pharmacy Technician by Jahangir Moini, published by Cengage Learning, Mar 3, 2010

22. Sundberg JA. Extemporaneous Compounding in the Hospital Pharmacy. International Journal of Pharmaceutical Compounding Issue: Sep/Oct 1997 Home Health Care URL:

http://www.ijpc.com/Products/ProductAddToCart.cf $m ? P I D=1263$

23. Cheryl A. Thompson; Recent FDA compounding guidances to pharmacies may not apply everywhere, American Journal of Health-System Pharmacy, Volume 75, Issue 5, 1 March 2018, Pages 258, https://doi.org/10.2146/news180015

24. Gigi D. Veterinary Compounding: Regulation, Challenges, and Resources Pharmaceutics. 2017 Mar; 9(1): 5. doi: [10.3390/pharmaceutics9010005] PMID: 28075379 
25. Schommer JC, Brown LM, Sogol EM. Section: Nuclear Pharmacy. Career Pathway Evaluation Program 2007 Pharmacist Profile Survey. June 2007.

26. SECTION .2700 - Nuclear Pharmacy URL: http://www.ncbop.org/LawsRules/rules.2700.pdf

27. APhA Home. Nuclear Pharmacy Practice. URL: https://pharmacist.com/nuclear-pharmacy-practice

28. Lam MS. Extemporaneous compounding of oral liquid dosage formulations and alternative drug delivery methods for anticancer drugs. Pharmacotherapy. 2011 Feb;31(2):164-92. doi: 10.1592/phco.31.2.164. Review. PubMed PMID: 21275495.

29. Mulla H, Tofeig M, Bu'Lock F, Samani N, Pandya HC. Variations in captopril formulations used to treat children with heart failure: a survey in the United kingdom. Arch Dis Child. 2007;92(5):409-11.

30. Special feature. Why specials manufacturing units are needed now as much as they ever were. The Pharmaceutical Journal (Vol 275) 17 December 2005, pp 143-144

31. Patel VP, Desai TR and others. EXTEMPORANEOUS DOSAGE FORM FOR ORAL LIQUIDS. Pharmacophore 2011, Vol. 2 (2), 86-103

32. Lopez FL, Ernest TB, Tuleu C, Gul MO. Formulation approaches to pediatric oral drug delivery: benefits and limitations of current platforms. Expert Opin Drug Deliv. 2015;12(11):1727-40.

33. Catherine M. Crill, Emily B. Hak, Lawrence A. Robinson, Richard A. Helms; Evaluation of microbial contamination associated with different $p$ methods for neonatal intravenous fat er infusion, American Journal of Health Pharmacy, Volume 67, Issue 11, 914-918, https://doi.org/10.2

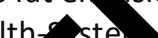
sion rat
39. Santoveña-Estévez A, Suárez-González J, Vera M, González-Martín C, Soriano M, Fariña JB. Effectiveness of Antimicrobial Preservation of Extemporaneous Diluted Simple Syrup Vehicles for Pediatrics. J Pediatr Pharmacol Ther. 2018;23(5):405409.

40. Guidance for Pharmacists on Extemporaneous Dispensing Pharmaceutical Society of Ireland Version 1 June 2015

41. Lowey A, Jackson M. How to ensure the quality and safety of unlicensed oral medicines. The Pharmaceutical Journal 29 August, 2008

42. Terrie YC. Pharmacy Compounding Is Flourishing Once Again. Pharmacy Times ${ }^{\circledR}$ November 01, 2005

43. Erickson MA. Your omm unding Questions Answered. Pha acy Time September 17, 2013

44. Weston SE Ketch KE. Ext mporaneous dispensing: a begin 's guide. In Whalley, Kate E Fletcher, SamE str, Rach l L Howard and Clare F wlinson. wund con in Pharmacy Practice Pu. ished by L, Pharmaceutical Press, 2008.

34. Francis J, Abraham S. Clinica Sharmacist Bridging the gap between patient and phrsicians. Soludi Pharm J. 2014;22(6):600

35. Goldman MP. Sodir tetra cyl sul for sclerotherapy tr atmel of ver s compounding pharmacy solk on sar h-matol Surg. 2004;30(12):145 doi: 10.1097/00042728200412010-00005.

36. Bai S, Dormer N, Shoults C, et al. Palatability of a novel oral formulation of prednisone in healthy young adults. J Pharm Pharmacol. 2017;69(4):489-496.

37. Gee SC, Hagemann TM. Palatability of liquid antiinfectives: clinician and student perceptions and practice outcomes. J Pediatr Pharmacol Ther. 2007;12(4):216-23.

38. Mistry P, Stirling H, Callens C, Hodson J, Batchelor H, SPaeDD-UK project. Evaluation of patient-reported outcome measurements as a reliable tool to measure acceptability of the taste of paediatric medicines in an inpatient paediatric population. BMJ Open. 2018;8(7):e021961. Published 2018 Jul 11. doi:10.1136/bmjopen-2018-021961 